\title{
Biogeochemical simulations to assess the impact of redox processes on mercury cycling in sediments
}

\author{
Stefanie HELMRICH ${ }^{1 *}$, DiMitRI Vlassopoulos ${ }^{2}$, \\ Charles N. AlPers ${ }^{3}$, Nigel W.T. QuinN ${ }^{4}$, Peggy A. \\ O'DAY ${ }^{1}$ \\ ${ }^{1}$ Univeristy of California Merced, Merced, CA, United States, \\ (*correspondence: shelmrich@ucmerced.edu, \\ oday@ucmerced.edu) \\ ${ }^{2}$ Anchor QEA LLC, Portland, OR, United States, \\ (dvlassopoulos@anchorqea.com) \\ ${ }^{3}$ U.S. Geological Survey, Sacramento, CA, United States, \\ (cnalpers@usgs.gov) \\ ${ }^{4}$ Lawrence Berkeley National Laboratory, Berkeley, CA, \\ United States (nwquinn@lbl.gov)
}

Methylmercury $(\mathrm{MeHg})$ in the environment poses a significant threat to human and ecological health and is a major contaminant of concern for water quality. Biogeochemical models that simulate mercury $(\mathrm{Hg})$ cycling in sediments are an emerging tool to aid management decisions. Such models can be used to assess driving processes and improve understanding of the complicated dynamics of $\mathrm{Hg}$ cycling. Redox cycling of sulfur (S) and iron $(\mathrm{Fe})$ exert strong controls on $\mathrm{Hg}$ cycling and net $\mathrm{MeHg}$ production, whereas manganese $(\mathrm{Mn})$ can be an indicator of redox conditions that are favorable for high $\mathrm{MeHg}$ production. A biogeochemical reaction model using the PHREEQC program was modified from prior studies to improve the model description of net $\mathrm{MeHg}$ production in sediments. The model connects $\mathrm{Hg}$ cycling with cycling of $\mathrm{Mn}, \mathrm{Fe}, \mathrm{S}$, and organic matter by considering primary and secondary redox reactions, adsorption-desorption, and mineral dissolution and precipitation. Rate equations to describe mercury methylation were coupled to biogeochemical redox reactions. Simulations were compared to chemical conditions in incubations of wetland sediments with known amounts of organic matter and electron acceptors, which allowed rates of biogeochemical processes to be estimated. Subsequent comparison of simulations to field data collected in reconnaissance monitoring of wetlands in the Grasslands Ecological Area in Merced County, CA was used to estimate transport processes within wetland sediments. Simulations showed that $\mathrm{Hg}$ speciation and solubility were controlled by $\mathrm{Fe}$ and $\mathrm{S}$ cycling in addition to microbial $\mathrm{Fe}(\mathrm{III})$ and sulfate reduction being driving forces for $\mathrm{Hg}$ methylation. This shows the importance of coupling between $\mathrm{Hg}$ and redox-sensitive element cycles for predicting Hg fate. 